\title{
Geostatistical inference using crosshole ground-penetrating radar
}

Looms, Majken C; Hansen, Thomas Mejer; Cordua, Knud Skou; Nielsen, Lars; Jensen, Karsten H.; Binley, Andrew

\section{Published in:}

Geophysics

Link to article, DOI:

10.1190/1.3496001

Publication date:

2010

Document Version

Publisher's PDF, also known as Version of record

Link back to DTU Orbit

Citation (APA):

Looms, M. C., Hansen, T. M., Cordua, K. S., Nielsen, L., Jensen, K. H., \& Binley, A. (2010). Geostatistical inference using crosshole ground-penetrating radar. Geophysics, 75(6), J29-J41.

https://doi.org/10.1190/1.3496001

\section{General rights}

Copyright and moral rights for the publications made accessible in the public portal are retained by the authors and/or other copyright owners and it is a condition of accessing publications that users recognise and abide by the legal requirements associated with these rights.

- Users may download and print one copy of any publication from the public portal for the purpose of private study or research.

- You may not further distribute the material or use it for any profit-making activity or commercial gain

- You may freely distribute the URL identifying the publication in the public portal

If you believe that this document breaches copyright please contact us providing details, and we will remove access to the work immediately and investigate your claim 


\title{
Geostatistical inference using crosshole ground-penetrating radar
}

\author{
Majken C. Looms ${ }^{1}$, Thomas M. Hansen ${ }^{2}$, Knud S. Cordua ${ }^{2}$, Lars Nielsen ${ }^{1}$, Karsten H. Jensen ${ }^{1}$, \\ and Andrew Binley ${ }^{3}$
}

\begin{abstract}
High-resolution tomographic images obtained from crosshole geophysical measurements have the potential to provide valuable information about the geostatistical properties of unsaturated-zone hydrologic-state variables such as moisture content. Under drained or quasi-steady-state conditions, the moisture content will reflect the variation of the physical properties of the subsurface, which determine the flow patterns in the unsaturated zone. Deterministic least-squares inversion of crosshole groundpenetrating-radar (GPR) traveltimes result in smooth, minimumvariance estimates of the subsurface radar wave velocity structure, which may diminish the utility of these images for geostatistical inference. We have used a linearized stochastic inversion technique to infer the geostatistical properties of the subsurface radar wave velocity distribution using crosshole GPR traveltimes directly. Expanding on a previous study, we have determined that it is possible to obtain estimates of global variance and
\end{abstract}

mean velocity values of the subsurface as well as the correlation lengths describing the subsurface velocity structures. Accurate estimation of the global variance is crucial if stochastic realizations of the subsurface are used to evaluate the uncertainty of the inversion estimate. We have explored the full potential of the geostatistical inference method using several synthetic models of varying correlation structures and have tested the influence of different assumptions concerning the choice of covariance function and data noise level. In addition, we have tested the methodology on traveltime data collected at a field site in Denmark. There, inferred correlation structures indicate that structural differences exist between two areas located approximately $10 \mathrm{~m}$ apart, an observation confirmed by a GPR reflection profile. Furthermore, the inferred values of the subsurface global variance and the mean velocity have been corroborated with moisturecontent measurements, obtained gravimetrically from samples collected at the field site.

\section{INTRODUCTION}

Crosshole ground-penetrating-radar (GPR) tomography has the potential to produce high-spatial-resolution images of the electromagnetic (EM) wave velocity distribution of the subsurface (e.g., Hubbard et al., 1997; Eppstein and Dougherty, 1998; Binley et al., 2001; Alumbaugh et al., 2002). The EM wave velocity is directly related to the relative permittivity of a material; in the unsaturated zone, the relative permittivity is strongly influenced by the moisture content of the porous media (Topp et al., 1980). Crosshole GPR therefore enables an indirect estimate of the subsurface moisture content. As a result, crosshole GPR methods have been used extensively for hydrologic applications (Annan, 2005).

The high-resolution 2D tomographic images obtained from cross- hole GPR data provide information regarding the spatial correlation structures of the subsurface that could otherwise be obtained only through extensive invasive sampling. Under drained or quasisteady-state conditions where the hydrologic-state variables can be expected to represent the physical properties of the subsurface, the geostatistical information (i.e., correlation lengths and variability) may serve as input to stochastic hydrologic models that provide more reliable predictions of water flow and therefore also contaminant transport (Hubbard et al., 1999; Binley et al., 2004). However, the quantitative estimation of $2 \mathrm{D}$ moisture-content images from crosshole GPR traveltimes is still subject to uncertainties, mainly associated with the assumed petrophysical relationships (used to compute moisture content from relative permittivity), data uncertainty, and assumptions inherent in the applied inversion algorithms. Sim-

Manuscript received by the Editor 22 December 2009; revised manuscript received 29 June 2010; published online 29 October 2010.

${ }^{1}$ University of Copenhagen, Department of Geography and Geology, Copenhagen, Denmark. E-mail: mcl@geo.ku.dk; ln@geo.ku.dk; khj@geo.ku.dk.

${ }^{2}$ Formerly University of Copenhagen; presently Technical University of Denmark, Center for Energy Resources Engineering, Lyngby, Denmark. E-mail: thomas.mejer.hansen@gmail.com.

${ }^{3}$ Lancaster University, Lancaster Environment Centre, Lancaster, U. K. E-mail: a.binley@ lancaster.ac.uk.

(c) 2010 Society of Exploration Geophysicists. All rights reserved. 
plifying the forward problem (ray theory, straight-ray approximation), measurement errors, and choosing regularization method, acquisition geometry, and an a priori model influence wave-velocity distribution properties (Menke, 1989; Peterson, 2001; Kowalsky et al., 2005; Linde et al., 2006; Cordua et al., 2008; Hansen et al., 2008). Deterministic least-squares inversion algorithms (e.g., Eppstein and Dougherty, 1998; Alumbaugh et al., 2002) produce smooth minimum-variance estimates of the model parameters with varying resolution throughout the interborehole region and inversion artifacts, particularly in areas with limited ray coverage (Day-Lewis et al., 2005). As a consequence and based on the analysis of five synthetic models, Day-Lewis and Lane (2004) conclude that GPR-derived tomograms may have little utility for inferring subsurface geostatistical properties. To improve the inverse estimate, stochastic inversion approaches (e.g., Hansen et al., 2006; Gloaguen et al., 2007; Dafflon et al., 2009) or full-waveform inversion algorithms (Ernst et al., 2007) can be adopted.

Work by Hansen et al. (2008) introduces and explores a novel methodology to infer the parameters of the covariance model directly from crosshole GPR tomography traveltime data making use of linearized stochastic inversion. In our work, we refer to this methodology as data-driven ergodic inference. For a specific choice of a prior covariance model, Hansen et al. (2008) evaluate whether realizations of the posterior Gaussian probability density function (PDF) are likely realizations of the prior Gaussian PDF. A likelihood distribution for a range of different prior covariance models is thus estimated. In Hansen et al. (2008), data-driven ergodic inference is tested by inferring correlation lengths for one synthetic model and a real data example using simplified assumptions regarding the forward problem and an assumed known global mean and variance of the velocity distribution. These initial applications have shown promising results.

The objective of our study is to further evaluate and expand the methodology described in Hansen et al. (2008). To mimic a realistic sensitivity of the EM radar signal, we use full-waveform forward modeling of the EM wave propagation to calculate the synthetic data using the finite-difference time-domain algorithm of Irving and Knight (2006) instead of the simplified ray approximation in Hansen et al. (2008). Furthermore, we infer the global variance and mean velocity of the subsurface along with the correlation structure, enabling a full geostatistical inference using crosshole GPR data alone. In Hansen et al. (2008), the global variance and mean velocity of the subsurface are assumed to be known prior to the inference. The sensitivity of the inferred geostatistical properties toward various correlation structures, the expected noise level of the GPR data, and the choice of covariance function are also evaluated. Finally, we apply the approach to four cross sections collected in the unsaturated zone at a field site in Denmark and compare the obtained results to independent information achieved from core samples and a GPR reflection survey.

\section{METHODS}

We consider the following linear problem:

$$
\mathbf{d}=\mathbf{G m},
$$

where $\mathbf{d}$ is a vector containing all data values, $\mathbf{m}$ contains the subsurface model parameters, and $\mathbf{G}$ is a linear mapping matrix relating the data to the model parameters. This way of defining the forward prob- lem is classical and has been used in a range of studies (e.g., Tarantola, 1987; Menke, 1989).

Traditionally, in the case of crosshole GPR velocity tomography based on traveltimes, $\mathbf{d}$ are the first-arrival traveltimes picked from the recorded GPR waveforms, $\mathbf{m}$ are the slowness (inverse velocity) values of a grid of cells describing the subsurface between two boreholes, and $\mathbf{G}$ contains the distances that the different rays have traveled in the individual cells. Here, however, we choose to parameterize the linear inverse problem such that $\mathbf{m}$ are velocities, $\mathbf{d}$ are the average velocities based on the observed traveltimes and raypath lengths, and $\mathbf{G}$ is a modification of the sensitivity kernel for the slowness parameterization such that the rows of $\mathbf{G}$ sum to one. This allows us to directly infer Gaussian statistics on the distribution of velocities rather than slownesses.

The generalized solution to linear inverse Gaussian problems, where the prior information of the model-parameter distribution is described by a Gaussian PDF and where measurement errors are assumed to be Gaussian, is given by (Tarantola and Valette, 1982)

$$
\mathbf{m}^{\mathrm{est}}=\langle\mathbf{m}\rangle+\mathbf{C}_{m} \mathbf{G}^{T}\left[\mathbf{G C}_{m} \mathbf{G}^{T}+\mathbf{C}_{d}\right]^{-1}[\mathbf{d}-\mathbf{G}\langle\mathbf{m}\rangle]
$$

and

$$
\mathbf{C}_{m}{ }^{\text {est }}=\left(\mathbf{G}^{T} \mathbf{C}_{d}^{-1} \mathbf{G}+\mathbf{C}_{m}{ }^{-1}\right)^{-1},
$$

where $\langle\mathbf{m}\rangle$ and $\mathbf{C}_{m}$ are the prior mean and covariance of the subsurface Gaussian PDF, $\mathbf{C}_{d}$ is the data covariance, and $\mathbf{m}^{\text {est }}$ and $\mathbf{C}_{m}{ }^{\text {est }}$ are the mean and covariance of the Gaussian posterior PDF. In deterministic least-squares inversion, $\mathbf{m}^{\text {est }}$ is chosen as the solution to the inverse problem. Although this estimate is the model with maximum posteriori likelihood, it is also the minimum variance estimate, which means that it is the model from the posterior distribution with least spatial variability, having a lower variability than assumed a priori through $\mathbf{C}_{m}$. However, the solution to the inverse problem is the entire posterior PDF given in equations 2 and 3, i.e., $N\left(\mathbf{m}^{\text {est }}, \mathbf{C}_{m}\right.$ est $)$, and not just $\mathbf{m}^{\text {est }}$.

Choosing the appropriate prior model and data covariance matrices is a nontrivial task that will significantly affect the a posteriori PDF. In this paper, we assume uncorrelated Gaussian measurement errors in the synthetic tests. Correlated data errors are likely to be present at our field site, as investigated by Cordua et al. $(2008,2009)$, and will therefore be accounted for in the analyses of the real data. However, the main focus will be kept on the a priori model covariance matrix, which contains information describing the assumed spatial correlation structures of the subsurface. Traditionally in deterministic least-squares inversion, $\mathbf{C}_{m}$ is designed to stabilize an otherwise underdetermined problem by damping (Marquardt, 1970) and/or smoothing (Constable et al., 1987) the model parameters. However, $\mathbf{C}_{m}$ can also be used to add a priori knowledge of the subsurface geostatistical properties to constrain the inversion (Tarantola and Valette, 1982).

The effect on the inverse estimate of varying $\mathbf{C}_{m}$ is illustrated in Figure 1, where a synthetic data set calculated for a synthetic subsurface is inverted under different assumptions of correlation length, global variance, and covariance function. The synthetic subsurface is a sequential simulation of a spherical covariance function with vertical and horizontal correlation lengths $\left(H_{\min }, H_{\max }\right)=(2 \mathrm{~m}, 4 \mathrm{~m})$ and global mean and variance of $0.13 \mathrm{~m} / \mathrm{ns}$ and $0.0002(\mathrm{~m} / \mathrm{ns})^{2}$, respectively. The choice of $\mathbf{C}_{m}$ has little influence on the deterministic least-squares estimate (see row I), even when the a priori assumptions are varied over an order of magnitude. However, varying $\mathbf{C}_{m}$ 
greatly influences the realizations drawn from the posterior PDF, i.e., $N\left(\mathbf{m}^{\text {est }}, \mathbf{C}_{m}{ }^{\text {est }}\right)$ (row II). These results emphasize the importance of using the correct geostatistical information concerning the subsurface if stochastic realizations are used to evaluate the subsurface structures and variability (as suggested by Dafflon et al., 2009).

The importance of inferring the geostatistical properties of the subsurface is therefore twofold:

1) To produce reliable inversion results. This, in particular, is essential when producing multiple realizations from the posterior PDF, illustrated clearly in Figure 1.

2) To describe representative subsurface properties, also in unprobed areas, used in stochastic hydrologic models to predict subsurface flow and transport.

\section{Data-driven ergodic inference}

To avoid choosing a potentially erroneous covariance model for the inversion, Hansen et al. (2008) suggest a method to infer the parameters of the covariance model directly from crosshole GPR traveltime data, making use of linearized stochastic inversion. The inversion routine in their work solves linear and linearizable inverse Gaussian problems using a stochastic approach and combines the fields of inverse theory and geostatistics (combining least-squares inversion and sequential simulation) to create realizations of the subsurface that honor the a priori covariance model as well as the observed data. Traveltime data (described as volume average data) and point data (e.g., neutron probe estimates of moisture content) can be used to condition the simulations.

In Hansen et al. (2008), the ergodic behavior of multiple realizations is used to evaluate whether the assumed a priori covariance model is consistent with the collected data and hence to infer the covariance model properties of the sampled subsurface. The methodology, i.e., data-driven ergodic inference, is presented in Figure 2. The semivariogram is often considered instead of the covariance model in geostatistics. From this point on, we refer to the semivariogram interchangeably with the covariance model and the sill value interchangeably with the global variance velocity. For second-order stationary random functions, the semivariogram $\gamma(h)$ and the covariance $\mathbf{C}(h)$ are related through $\gamma(h)=\mathbf{C}(0)-\mathbf{C}(h)$, where $h$ is the distance or lag (Journel and Huijbregts, 1978).

Initially, an assumed covariance model (Figure 2a) is used as input in a sequential simulator to generate, in this case, 100 unconditional realizations of the subsurface. Because of the finite size of the model grid, ergodic fluctuations of the semivariogram (Figure 2b) will occur and can be described by a Gaussian distribution centered on the model semivariogram (Figure 2c). The linearized stochastic inversion produces a posterior PDF (Figure 2d) from which 100 conditioned realizations can be drawn (Figure 2e). A likelihood value can be computed (Figure 2f) for each of the realizations in Figure 2e to determine whether they are likely samples of the distribution in Figure 2c. Finally, the average likelihood of the 100 realizations is found, representing the probability that the prior covariance model is consistent with the data collected. The range of probable correlation

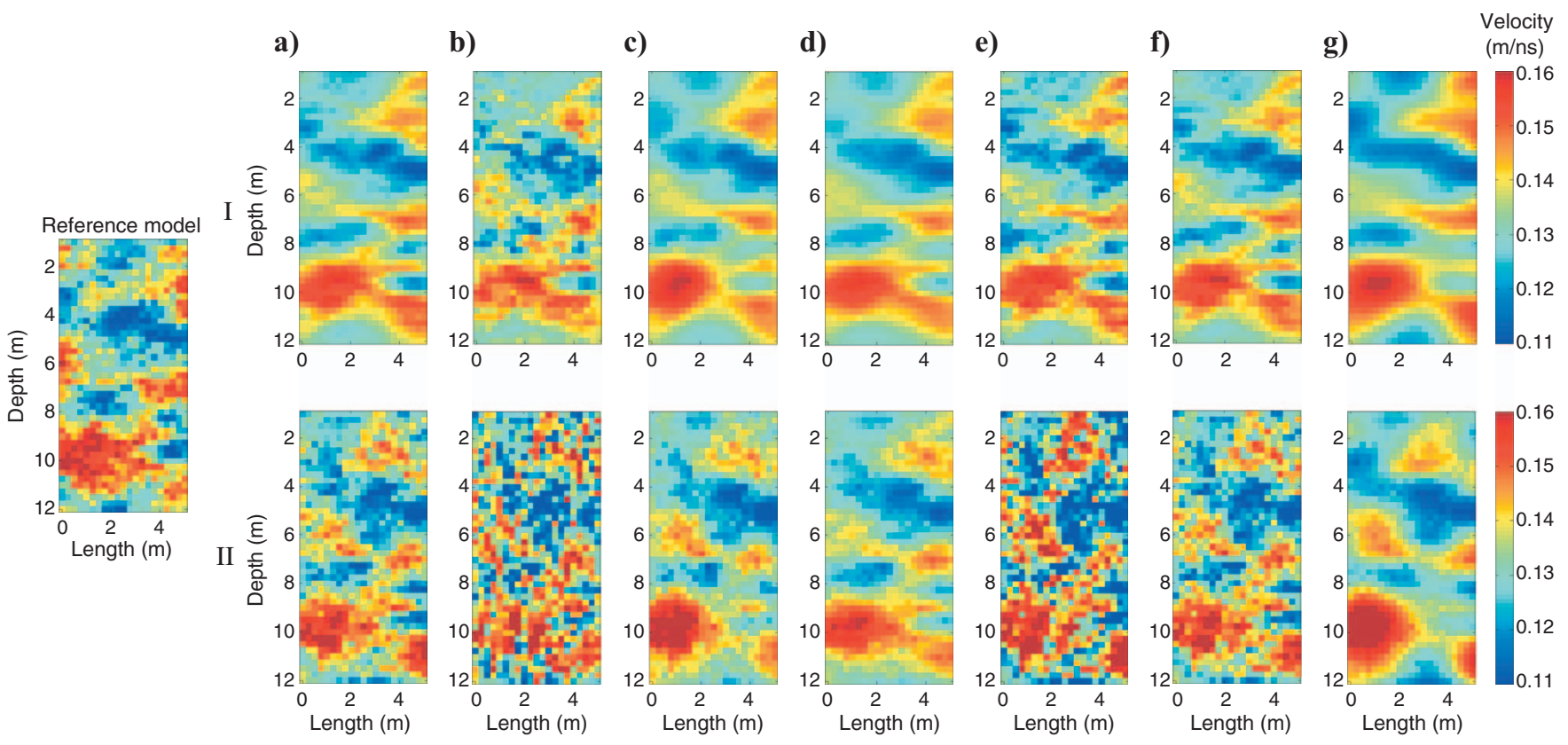

Figure 1. The importance of the chosen a priori covariance matrix $\mathbf{C}_{m}$ on the inversion estimate. The true subsurface velocity structure is shown to the left. The top row (I) is the least-squares inversion estimates, and the lower row (II) is selected realizations drawn from the posterior PDF, shown for six assumptions regarding the model $\mathbf{C}_{m}$ : (a) true values - spherical $\mathbf{C}_{m}$ with $H_{\min }, H_{\max }=(2 \mathrm{~m}, 4 \mathrm{~m})$ and $\sigma^{2}=0.0002(\mathrm{~m} / \mathrm{ns})^{2}$; (b) low correlation length - spherical $\mathbf{C}_{m}$ with $H_{\min }, H_{\max }=(0.5 \mathrm{~m}, 0.5 \mathrm{~m})$ and $\sigma^{2}=0.0002(\mathrm{~m} / \mathrm{ns})^{2}$; (c) high correlation length - spherical $\mathbf{C}_{m}$ with $H_{\min }, H_{\max }=(10 \mathrm{~m}, 10 \mathrm{~m})$ and $\sigma^{2}=0.0002(\mathrm{~m} / \mathrm{ns})^{2}$; (d) low variance - spherical $\mathbf{C}_{m}$ with $H_{\min }, H_{\max }=(2 \mathrm{~m}, 4 \mathrm{~m})$ and $\sigma^{2}$ $=0.00002(\mathrm{~m} / \mathrm{ns})^{2} ;(\mathrm{e})$ high variance - spherical $\mathbf{C}_{m}$ with $H_{\min }, H_{\max }=(2 \mathrm{~m}, 4 \mathrm{~m})$ and $\sigma^{2}=0.001(\mathrm{~m} / \mathrm{ns})^{2}$; (f) exponential $\mathbf{C}_{m}$ with $H_{\min }$, $H_{\max }=(2 \mathrm{~m}, 4 \mathrm{~m})$ and $\sigma^{2}=0.0002(\mathrm{~m} / \mathrm{ns})^{2}$; and $(\mathrm{g})$ Gaussian $\mathbf{C}_{m}$ with $H_{\min }, H_{\max }=(2 \mathrm{~m}, 4 \mathrm{~m})$ and $\sigma^{2}=0.0002(\mathrm{~m} / \mathrm{ns})^{2}$. 
lengths, describing the spatial structures of the subsurface, can thereby be inferred indirectly by exploring a parameter space of covariance models.

For a more in-depth description of how the prior covariance properties are inferred and the mathematical description of the inversion routine, we refer to Hansen et al. (2006), Hansen and Mosegaard (2008), and Hansen et al. (2008). In our current study, the forward calculation of traveltimes is based on the finite-difference solution to the eikonal equation described by Zelt and Barton (1998).

Figure 3 illustrates the principle behind data-driven ergodic inference for the results presented in Figure 1. In Figure 3, the experimental semivariograms of 100 posterior realizations (gray) are compared to the $95 \%$ confidence intervals of 100 a priori realizations (red) for the seven different choices of covariance models presented in Figure 1. If we have made appropriate a priori assumptions concerning the covariance model, the 100 posterior realizations should fall within the range of the a priori realizations, resulting in a high likelihood value. This is the case when the spherical (true reference model), exponential, and Gaussian covariance functions are used as prior information (Figure 3a, f, and g, respectively). The proposed methodology therefore appears to provide limited information on the covariance model type. However, assuming an erroneous correlation structure results in posterior semivariograms that differ substantially from the a priori semivariograms (Figure $3 \mathrm{~b}$ and c), and assuming a too low global a priori variance produces posterior realizations with substantially higher sill values (Figure 3d), and vice versa (Figure 3e). We therefore expect to be able to estimate the correct global variance and correlation structure using data-driven ergodic inference.

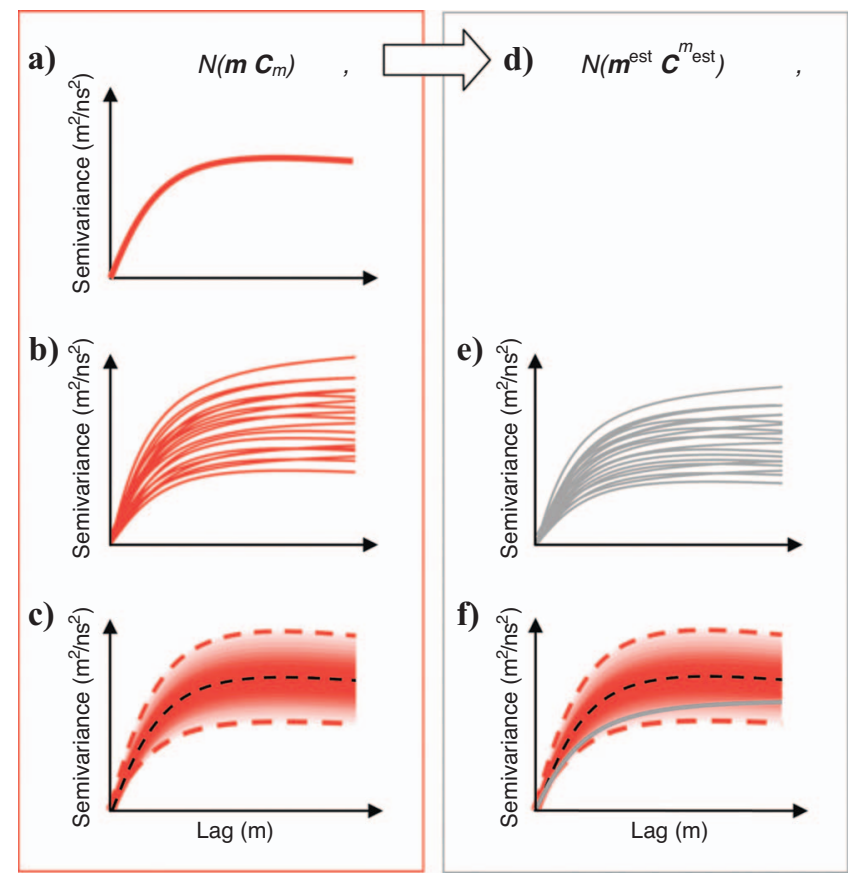

Figure 2. Schematic of the geostatistical inference using the proposed methodology, i.e., data-driven ergodic inference. See text for detailed description. (a) The a priori model; (b) 100 unconditioned realizations; (c) likelihood distribution; (d) a posteriori model; (e) 100 conditioned realizations; (f) likelihood value.

\section{SYNTHETIC TESTS}

Synthetic case studies, with varying covariance models, are examined to explore the limitations and advantages of using data-driven ergodic inference. Twenty unconditioned realizations of four covariance models (referred to as models I, II, III, and IV) -80 in all - with vertical and horizontal correlation lengths $\left(H_{\min }, H_{\max }\right)$ $=(1 \mathrm{~m}, 2 \mathrm{~m}),(2 \mathrm{~m}, 4 \mathrm{~m}),(4 \mathrm{~m}, 8 \mathrm{~m})$, and $(5 \mathrm{~m}, 1.5 \mathrm{~m})$ are used as synthetic representations of the subsurface. The four covariance models chosen are, in the following, referred to as the reference covariance models. We distinguish between the covariance models of the reference models and the covariance models of 80 realizations because the covariance models of the individual realizations are not always identical to the reference covariance models as a result of ergodic fluctuations (illustrated in Figures 5 and 6).

The synthetic tests are set up to closely resemble the case study examined later with a model domain extending $5 \mathrm{~m}$ horizontally and $12 \mathrm{~m}$ vertically. A Gaussian velocity distribution is used with a mean velocity and variance set to $0.13 \mathrm{~m} / \mathrm{ns}$ and $0.0002 \mathrm{~m}^{2} / \mathrm{ns}^{2}$, respectively. Spherical covariance models, with a higher correlation in the horizontal direction, were chosen because they are believed to capture the heterogeneous characteristics of the sedimentary deposits at the field study area (Hansen et al., 2008). However, model IV, which has a higher vertical correlation, is also included to test the limitations of the methodology. This model is not considered to be a likely representation of the geologic environment at the study area.

Synthetic EM waveforms are calculated for the 744 antenna positions, described in the data acquisition section of the case study, using a full-waveform finite-difference time-domain modeling algorithm (Irving and Knight, 2006). A Blackman-Harris pulse with a central frequency of $100 \mathrm{MHz}$ is used, and the antennas are approximated with vertical, infinitesimal dipoles. The EM wave velocities of the chosen realization are transformed into dielectric permittivity using a high-frequency approximation (e.g., Ernst et al., 2006), and the electric conductivity is set to $2 \mathrm{mS} / \mathrm{m}$ for all model cells representing the average conductivity at the case study field site at Arrenæs, Denmark (cf. Looms et al., 2008). The relative magnetic permeability is set to one, given that near-surface geologic materials are generally nonmagnetic (e.g., Davis and Annan, 1989). The first-arrival traveltime data are found with a semiautomatic picking routine used also for the field data. White random noise with a standard deviation of $0.4 \mathrm{~ns}$ is added to the traveltime data. Unless otherwise stated, the noisy synthetic data set is used as input in the inversion, assuming the correct noise level with a standard deviation of $0.4 \mathrm{~ns}$.

\section{Inference of global variance and mean velocity}

If a 2D setup is considered, six parameters that describe the a priori choice of a Gaussian PDF can be varied: type of covariance model, $H_{\min }, H_{\max }$, dip, global variance, and mean velocity. As many of the unknown parameters as possible should be estimated prior to the inversion using other independent information. In our study, such information is assumed to be unavailable; therefore, we attempt to estimate the global variance and mean using only the traveltime data collected.

The synthetic traveltime data from the 80 realizations detailed above are converted to velocity data, assuming that the EM wave has traveled the shortest geometric distance between the transmitter and the receiver. The mean and variance of the traveltime velocity data are subsequently compared with the true mean and variance velocity values. Conversion factors between true and estimated values (e.g., 
$\left.\sigma_{\text {true }}^{2} / \sigma_{\text {est }}^{2}\right)$ are determined and tabulated in Table 1 . The average velocity estimates, computed from the synthetic traveltimes, are a good measure to determine the true mean velocity of the subsurface, varying only up to $3 \%$ from the true average velocity. However, the variance estimates cannot be converted to the true variance values of the subsurface in the same straightforward manner. This can be ascribed to averaging the velocity as the wave passes through the subsurface due to the sensitivity of the EM waves. The conversion factors for the variance estimates in Table 1 depend highly on the reference covariance model and the selected realization, varying from 1.12 to 26.04 with an average of 6.13 . The covariance model with largest horizontal correlation lengths (model III) generally results in a better estimate of the subsurface variance because the velocity structures are larger and therefore better captured by the passing rays. Contrary to this, small-scale variability (model I) and variability in the vertical direction (model IV) become difficult to resolve using crosshole GPR because of the physical extent of the sensitivity kernel of the considered GPR frequency (i.e., $100 \mathrm{MHz}$ ) and the dominating horizontal nature of the measurement scheme, respectively.

Next, we estimate the global variance using data-driven ergodic inference by varying the assumed global variance and determining the corresponding likelihood values. Conversion factors assuming known (i.e., $H_{\min }$ and $H_{\max }$ are set equal to the reference covariance models) and unknown (i.e., $H_{\min }$ and $H_{\max }$ vary from 0.01 to $10 \mathrm{~m}$ ) subsurface correlation structures in the inversion are tabulated in Table 2. Four examples of estimating the global variance, representing the four different correlations structures, are presented in Figure 4.

From Figure 4, it is apparent that the global variance can be estimated with a high accuracy because the likelihood values of testing different levels of a priori variances have a well-defined peak near the correct variance value. This is also seen in Table 2 , where the conversion factors show that the global variance of the subsurface can be recovered with $\pm 50 \%$ accuracy if the subsurface correlation structures are known and within a factor of two if the subsurface structures are unknown.

\section{Inference of correlation structure}

Next, we want to estimate the subsurface correlation structures. First, we assume that the global variance of the subsurface is known. The correlation lengths are sampled with $H_{\min }$ from 0.01 to $10 \mathrm{~m}$ and $H_{\max }$ from 0.02 to $10 \mathrm{~m}$. For each covariance model, a likelihood value is estimated following the schematic approach shown in Figure 2. Likelihood distribution plots of five models representing each reference covariance model are presented in Figure 5.
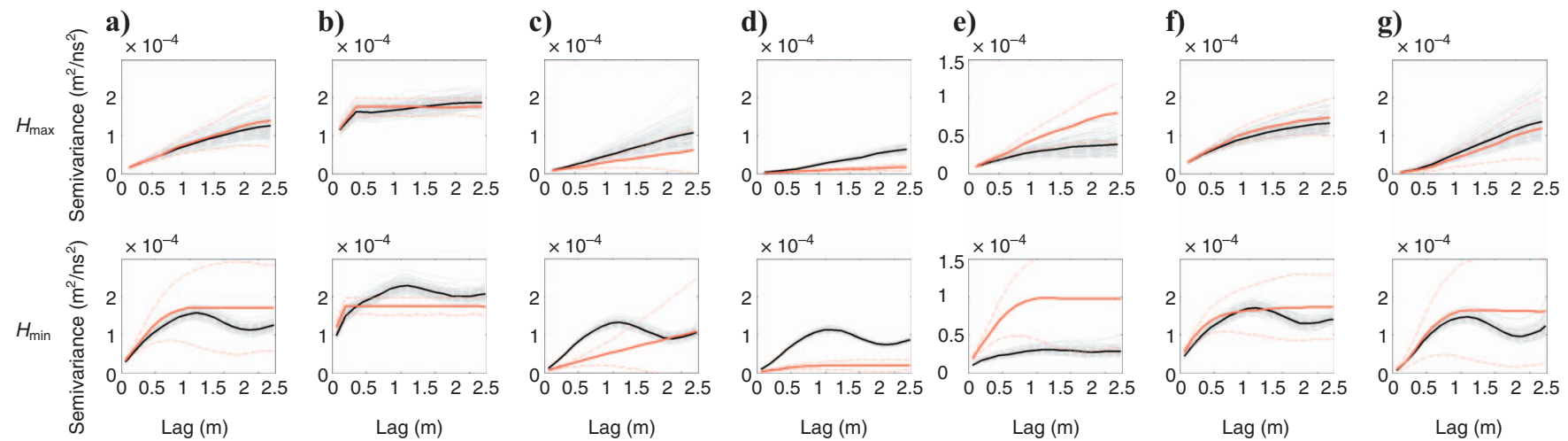

Figure 3. The importance of the chosen a priori covariance matrix $\mathbf{C}_{m}$ on the experimental semivariograms. Semivariograms in the (top row) horizontal $H_{\min }$ and (bottom row) vertical $H_{\max }$ directions for six assumptions regarding model $\mathbf{C}_{m}$ : (a) true values - spherical $\mathbf{C}_{m}$ with $H_{\min }, H_{\max }$ $=(2 \mathrm{~m}, 4 \mathrm{~m})$ and $\sigma^{2}=0.0002(\mathrm{~m} / \mathrm{ns})^{2} ;(\mathrm{b})$ low correlation length - spherical $\mathbf{C}_{m}$ with $H_{\min }, H_{\max }=(0.5 \mathrm{~m}, 0.5 \mathrm{~m})$ and $\sigma^{2}=0.0002(\mathrm{~m} / \mathrm{ns})^{2}$; (c) high correlation length - spherical $\mathbf{C}_{m}$ with $H_{\min }, H_{\max }=(10 \mathrm{~m}, 10 \mathrm{~m})$ and $\sigma^{2}=0.0002(\mathrm{~m} / \mathrm{ns})^{2}$; (d) low variance - spherical $\mathbf{C}_{m}$ with $H_{\min }$, $H_{\max }=(2 \mathrm{~m}, 4 \mathrm{~m})$ and $\sigma^{2}=0.00002(\mathrm{~m} / \mathrm{ns})^{2}$; (e) high variance - spherical $\mathbf{C}_{m}$ with $H_{\min }, H_{\max }=(2 \mathrm{~m}, 4 \mathrm{~m})$ and $\sigma^{2}=0.001(\mathrm{~m} / \mathrm{ns})^{2}$; (f) exponential $\mathbf{C}_{m}$ with $H_{\min }, H_{\max }=(2 \mathrm{~m}, 4 \mathrm{~m})$ and $\sigma^{2}=0.0002(\mathrm{~m} / \mathrm{ns})^{2}$; and $(\mathrm{g})$ Gaussian $\mathbf{C}_{m}$ with $H_{\min }, H_{\max }=(2 \mathrm{~m}, 4 \mathrm{~m})$ and $\sigma^{2}=0.0002(\mathrm{~m} / \mathrm{ns})^{2}$. The red lines show the average and $95 \%$ confidence interval of experimental semivariograms computed for the 100 unconditioned realizations; the gray lines are the experimental semivariograms of the 100 conditioned realizations with the average shown in black. The $y$-axis range of (e) differs from the rest.

Table 1. Mean velocity and variance conversion factors using traveltime data.

\begin{tabular}{|c|c|c|c|c|c|c|}
\hline \multirow[b]{2}{*}{ Model } & \multicolumn{3}{|c|}{ Velocity } & \multicolumn{3}{|c|}{ Variance } \\
\hline & Mean & Minimum & Maximum & Mean & Minimum & Maximum \\
\hline I & 0.98 & 0.97 & 1.00 & 7.13 & 3.92 & 11.31 \\
\hline II & 0.99 & 0.97 & 1.02 & 4.13 & 1.76 & 7.76 \\
\hline III & 1.00 & 0.97 & 1.03 & 3.34 & 1.12 & 8.53 \\
\hline IV & 1.01 & 0.98 & 1.03 & 9.93 & 2.49 & 26.04 \\
\hline Mean & 0.99 & 0.97 & 1.02 & 6.13 & 2.32 & 13.41 \\
\hline
\end{tabular}


As expected in Figure 5, the prior covariance models having the highest likelihood (yellow circles) are not identical to the reference prior covariance models used to create the images (red circles). The observed discrepancies are caused in part by the ergodic fluctuations since the covariance model inferred from the chosen realization (green circles) also varies from the reference model. This tendency is most pronounced as the correlation lengths tend toward and exceed the model size (Hansen et al., 2008). The ergodic variations also increase the uncertainty bounds of the most likely prior covariance model. This characteristic is very clear for models III and IV $\left[\left(H_{\min }, H_{\max }\right)=(4 \mathrm{~m}, 8 \mathrm{~m})\right.$ and $(5 \mathrm{~m}, 1.5 \mathrm{~m})$, respectively $]$, where the ranges of correlation lengths with high likelihood (dark gray areas) increase considerably more than those of model I, i.e., $\left(H_{\min }, H_{\max }\right)=(1 \mathrm{~m}, 2 \mathrm{~m})$.

Figure $6 \mathrm{c}$ summarizes the result of estimating the most likely correlation length for the 80 tested models, assuming the global variance is known. For comparison, three other results are included in the figure. Figure $6 \mathrm{a}$ presents the correlation structures obtained through a least-squares fit of the experimental semivariogram when the subsurface is known (the green dots in Figure 5). This estimate is comparable to a standard geostatistical inference using e.g., Gstat
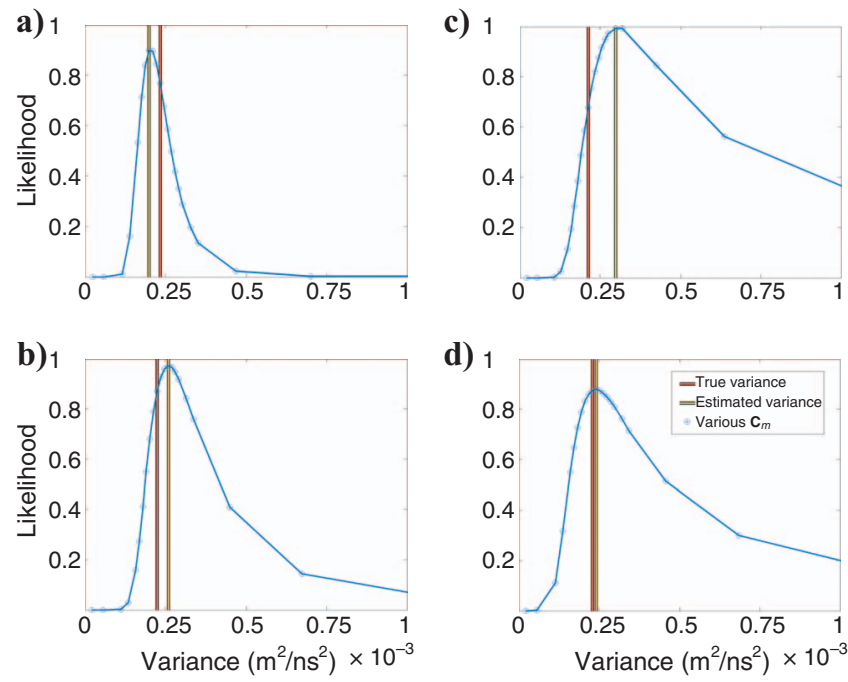

Figure 4. Estimate of the global variance using data-driven ergodic inference. The likelihoods of a range of prior global variances are shown with blue; the global variance with the highest likelihood and the true global variance are indicated with yellow and red, respectively. Examples are shown for (a) model I, (b) model II, (c) model III, and (d) model IV. For each model, the inference of the first synthetic representations of the subsurface is shown.
(Pebesma and Wesseling, 1998). In Figure 6b, the subsurface is also assumed to be known, but the geostatistical inference is obtained using data drive ergodic inference. In this particular case, we use the experimental semivariogram in Figure 6a and estimate the most likely covariance model that describes this semivariogram. Note that the results in Figure 6a and $\mathrm{b}$ represent the ideal case where the subsurface model is known, and thus no inversion is needed. Finally, in Figure $6 \mathrm{~d}$, we include the result of estimating the most likely correlation lengths within the data-driven ergodic inference framework using the global variance estimated above, assuming that the correlation lengths were unknown (see Table 2). This last result corresponds to a situation where no prior knowledge concerning the subsurface structures is known, perhaps the most common scenario.

As mentioned, Figure 6a illustrates how ergodic fluctuations cause the covariance models of the individual realizations to differ from the reference covariance models. The geostatistical inference of the correlation structures becomes increasingly challenging when the structures exceed the model bounds in which case the experimental semivariograms do not reach their sill value. Here, we have even aided the inference by assuming the sill value is known. Using data-driven ergodic inference to estimate the most likely covariance model in Figure $6 \mathrm{~b}$ improves the recoverability of the reference model, i.e., the variability of the estimated $4 \times 20$ covariance models are narrower compared to Figure 6a. It may be somewhat surprising that of the methods considered here, the best inference is obtained using data-driven ergodic inference. However, most traditional methods used for spatial inference based on regression analysis (e.g., Pebesma and Wesseling, 1998) do not account for the ergodic nature of the semivariogram, where the variability of the semivariance will be larger for larger offset than smaller offset and where the variability increases as the ranges increase as we have discussed. For point-based data, maximum-likelihood estimation is an alternative that by nature considers ergodic variability (Pardo-Igúzquiza, 1998).

Figure $6 \mathrm{~b}$ represents the best obtainable inference results possible using data-driven ergodic inference and could only be achieved if the collected GPR data set were error free, with a perfect resolution of the subsurface (i.e., a well-determined problem) and correct assumptions concerning the inverse problem. For this ideal case, we still observe that the variability from the reference model increases for larger correlation structures.

An overall good estimation of the geostatistical properties is observed in Figure $6 \mathrm{c}$ and d. In fact, the data-driven ergodic inference using only crosshole GPR data almost recovers the true geostatistical properties of the subsurface as well as direct inference using conventional methods when the subsurface structure is known (Figure

Table 2. Variance conversion factors using data-driven ergodic inference.

\begin{tabular}{|c|c|c|c|c|c|c|}
\hline \multirow[b]{2}{*}{ Model } & \multicolumn{3}{|c|}{ Known correlation structures } & \multicolumn{3}{|c|}{ Unknown correlation structures } \\
\hline & Mean & Minimum & Maximum & Mean & Minimum & Maximum \\
\hline I & 0.89 & 0.70 & 1.15 & 0.80 & 0.49 & 1.15 \\
\hline II & 1.00 & 0.60 & 1.25 & 1.07 & 0.72 & 1.49 \\
\hline III & 1.15 & 0.70 & 1.50 & 1.30 & 0.78 & 2.31 \\
\hline IV & 1.05 & 0.60 & 1.40 & 0.74 & 0.41 & 1.11 \\
\hline Mean & 1.02 & 0.65 & 1.33 & 0.98 & 0.60 & 1.52 \\
\hline
\end{tabular}


6a). The vertical correlation lengths $H_{\min }$ are particularly well determined. This is partly the result of the measurement scheme using mainly horizontal or quasi-horizontal rays. A good vertical resolution is thus achieved, although the horizontal changes are more difficult to capture. The correlation lengths in the vertical direction are, in models I-III, also smaller than the model bounds, enabling a better inference in this direction. The horizontal correlation lengths are, for most realizations, also inferred reasonably well - even for model III, where the horizontal correlation length $\left(H_{\max }=8 \mathrm{~m}\right)$ exceeds the model domain of $5 \mathrm{~m}$. Note that the uncertainty of the inference of model I (Figure 6c and d) is not as small as in Figure 6b. We assume that the EM waves can be described by bended rays in the inversion procedure, and therefore the true physical sensitivity of the synthetic data obtained using a full-waveform forward solver cannot be accounted for. This effect is most pronounced in models with small correlation structures.

\section{Effect of noise}

In the above analyses, the noise added to the synthetic data had a standard deviation of $0.4 \mathrm{~ns}$ and we assumed the correct noise level in the inversion. Figure 7 illustrates the effect of adding six different noise levels $(0.1,0.2,0.4,0.6,0.8$, and $1.2 \mathrm{~ns})$ to the traveltime data of model Ia (Figure 5). The increased noise level is also accounted for in the inversion by increasing the expectation to the data-error variance along the diagonal in the data covariance matrix (equations 2 and 3 ).

The estimated likelihood plots in Figure 7 clearly show an effect of the noise added. Note that the altered data noise is accounted for in the inversion. The range of prior covariance models with high likelihood increases as the noise increases. In other words, the confidence bounds of the most likely prior model are increased. Furthermore, a)
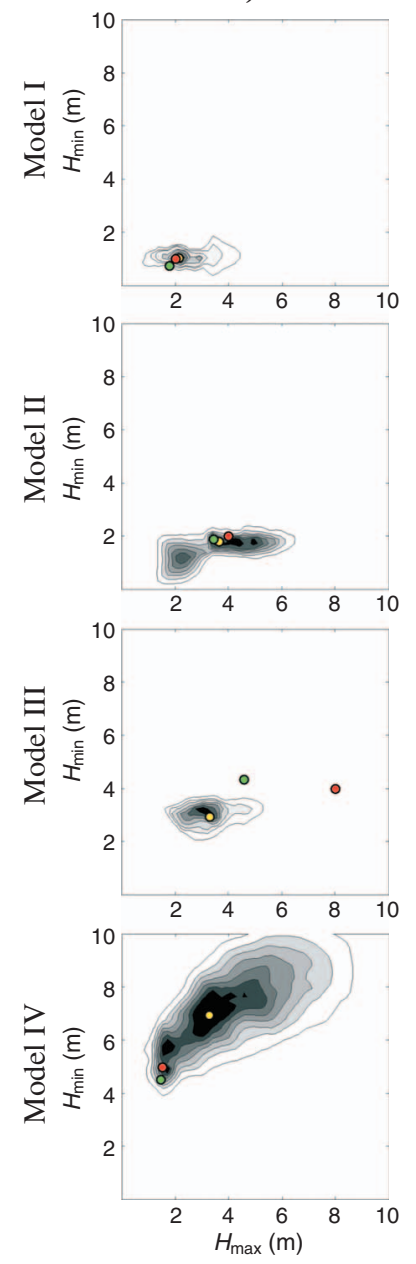

b)
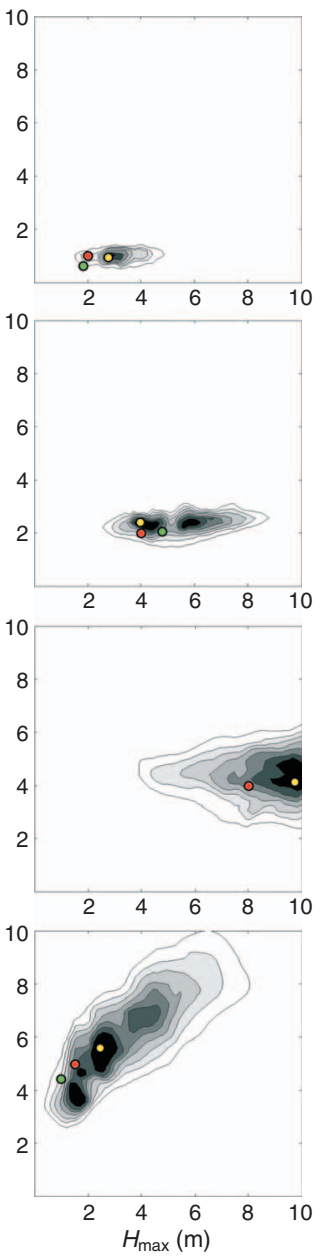

c)
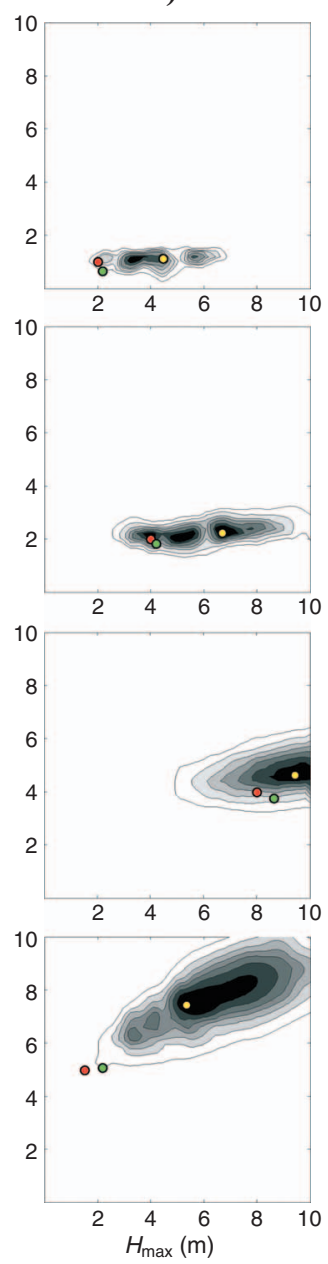

d)
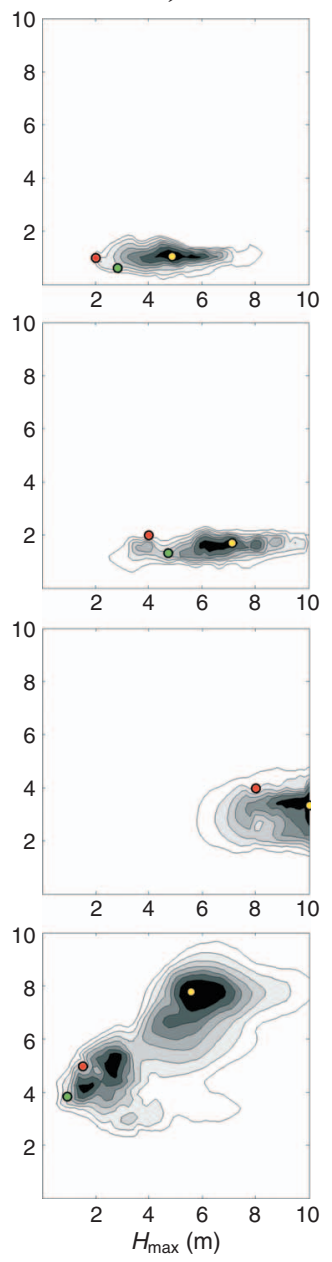

e)
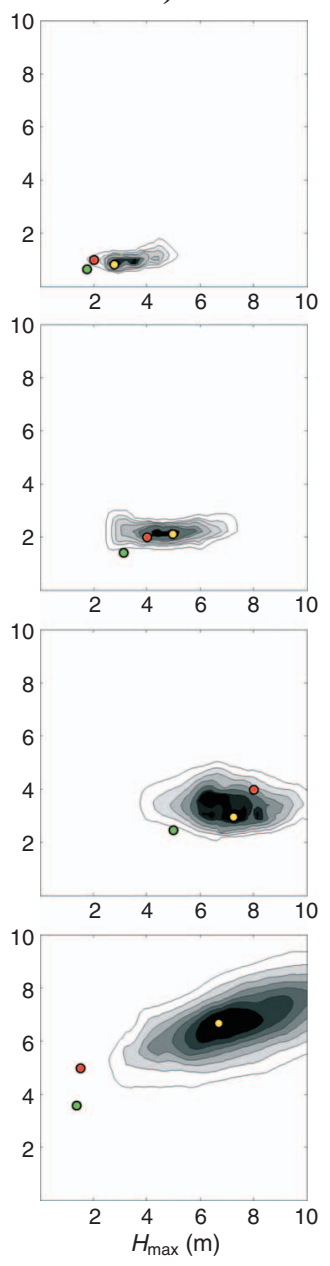

Figure 5. Contour plots showing the likelihood as a function of $H_{\min }$ and $H_{\max }$ for models I-IV obtained using data-driven ergodic inference. (a-e). Likelihood plots for the five first synthetic representations of the four models with varying subsurface structure. The reference and most likely prior covariance models are marked with a red and yellow dots, respectively, whereas the geostatistical inference, obtained through a least-squares fit of the experimental semivariogram, is marked with a green dot. 

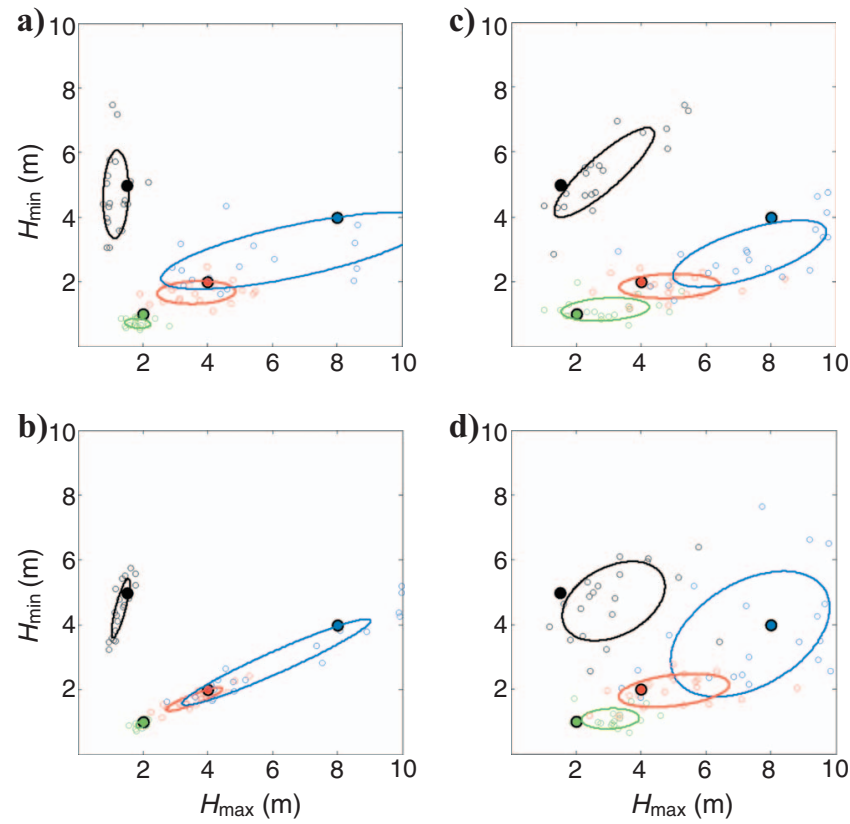

Figure 6. The geostatistical inference of the 80 synthetic representations of the subsurface. Results for models I-IV are shown with green, red, blue, and black colors, respectively. The circles illustrate the respective ranges of a 2D Gaussian fit to the 20 estimated $H_{\min } / H_{\max }$ values. (a) Geostatistical inference, obtained through a least-squares fit of the experimental semivariogram when the subsurface is known. (b) Data-driven ergodic inference when the subsurface is known. (c) Data-driven ergodic inference for crosshole GPR data, assuming a known global variance. (d) Data-driven ergodic inference for crosshole GPR data using the global variance estimated within the methodology. the most likely $H_{\max }$ value increases at a high noise level, from between 2.13 and $2.70 \mathrm{~m}$ at $0.1-0.8 \mathrm{~ns}$ to $6.01 \mathrm{~m}$ at $1.2 \mathrm{~ns}$. However, the $H_{\min }$ values with maximum likelihood are largely unaffected, varying from 0.88 to $1.36 \mathrm{~m}$. These results imply that the data-driven ergodic inference suffers greatly at very large noise levels. Low noise contamination of the data, around $0.4 \mathrm{~ns}$ and below $0.8 \mathrm{~ns}$, should therefore be maintained.

\section{Effect of different covariance functions}

The result of inferring the correlation structures of model IIa (Figure 5), assuming spherical, exponential, and Gaussian covariance functions, is shown in Figure 8. The inferred most likely correlation lengths vary according to the different covariance functions, which is expected because the correlation lengths or ranges do not represent the same subsurface variability (Goovaerts, 1997). Nonetheless, we obtain quite similar least-squares estimates and realizations when we use the inferred most likely correlation structures obtained in Figure 8 in an inversion (see Figure 9a and b, respectively). The exponential and spherical realizations are almost identical, but the Gaussian realization differs slightly, most probably the effect of the substantially different shape of the Gaussian semivariogram at short lags. One could argue that the proposed methodology infers correlation structures that honor the collected traveltime data independent of the chosen covariance function. As a result, a priori knowledge of the covariance function is less crucial, as one would expect, given that the inversion results (i.e., $\mathbf{m}^{\text {est }}$ and the stochastic realizations) do not suffer adversely. This is especially true for the exponential and spherical covariance models.

The likelihood values and data misfit value obtained using the most likely covariance models are presented in Table 3 . For the data set examined, there appears to be a slight indication that the spherical covariance function (on which the reference model is based) results in the most likely fit for the chosen data, represented by the highest likelihood value and the lowest data misfit. However, it is not yet certain whether this difference is sufficient to make a unique estimation.

\section{CASE STUDY}

\section{Field site}

A field site was established in Arrenæs, Denmark, on a 20-30-m deposit of unsaturated alluvial sand sediments. A schematic of the field-site setup is illustrated in Figure 10. The experimental setup consists of eight boreholes drilled to a depth of $12 \mathrm{~m}$, with PVC access tubes for GPR antennas installed. The eight boreholes are divided into two separate groups, each group having four boreholes forming a cross consisting of two 5-m-long lines. The two groups are approximately $10 \mathrm{~m}$ apart along a north-south transect.

Grain-size analyses of sediment samples taken in a 14.5-m-deep cored borehole within area B (blue circle, Figure 10) indicate that the top $1 \mathrm{~m}$ (approximately) consists of loam and clay. Below this topsoil, fine and coarse sand is found, with thin intercalations of silt (approximately 8-m 
depth) and clay (approximately 10-m depth) layers. Gravimetric measurements of moisture content were obtained in up to 25 -cm-thick sediment samples throughout the depth of the cored borehole. The mean and variance of the moisture content measurements from 1 to $12 \mathrm{~m}$ were 0.058 and 0.0012 , respectively. These values correspond to mean and variance EM wave velocities of $0.1488 \mathrm{~m} / \mathrm{ns}$ and $0.00025 \mathrm{~m}^{2} / \mathrm{ns}^{2}$ using the modified Topp's equation (Ferré et al., 1996).

\section{Data acquisition}

The crosshole GPR measurements were conducted using a Sensors \& Software PulseEKKO PE100 system equipped with 100$\mathrm{MHz}$ borehole antennas.
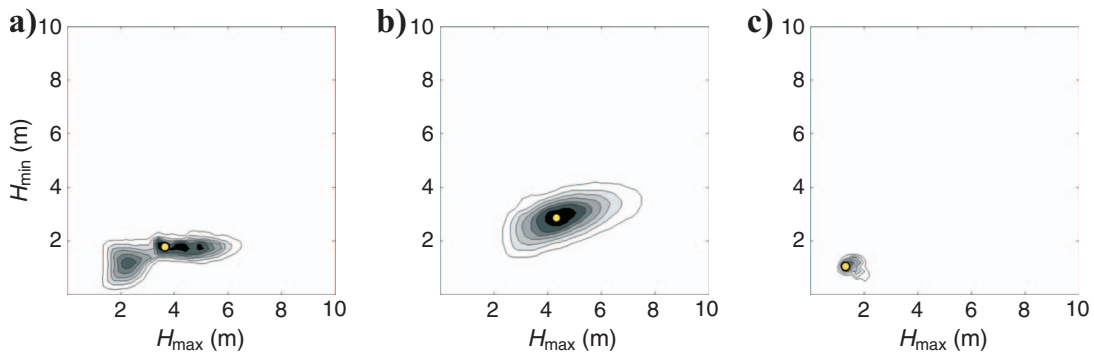

Figure 8. The effect of assuming different covariance model types on the data-driven ergodic inference result. Contour plots show the likelihood as a function of $H_{\min }$ and $H_{\max }$. Model IIa (Figure 5) is shown as an example. (a) Spherical model, (b) exponential model, and (c) Gaussian covariance model. The most likely prior covariance model is marked with a yellow dot.
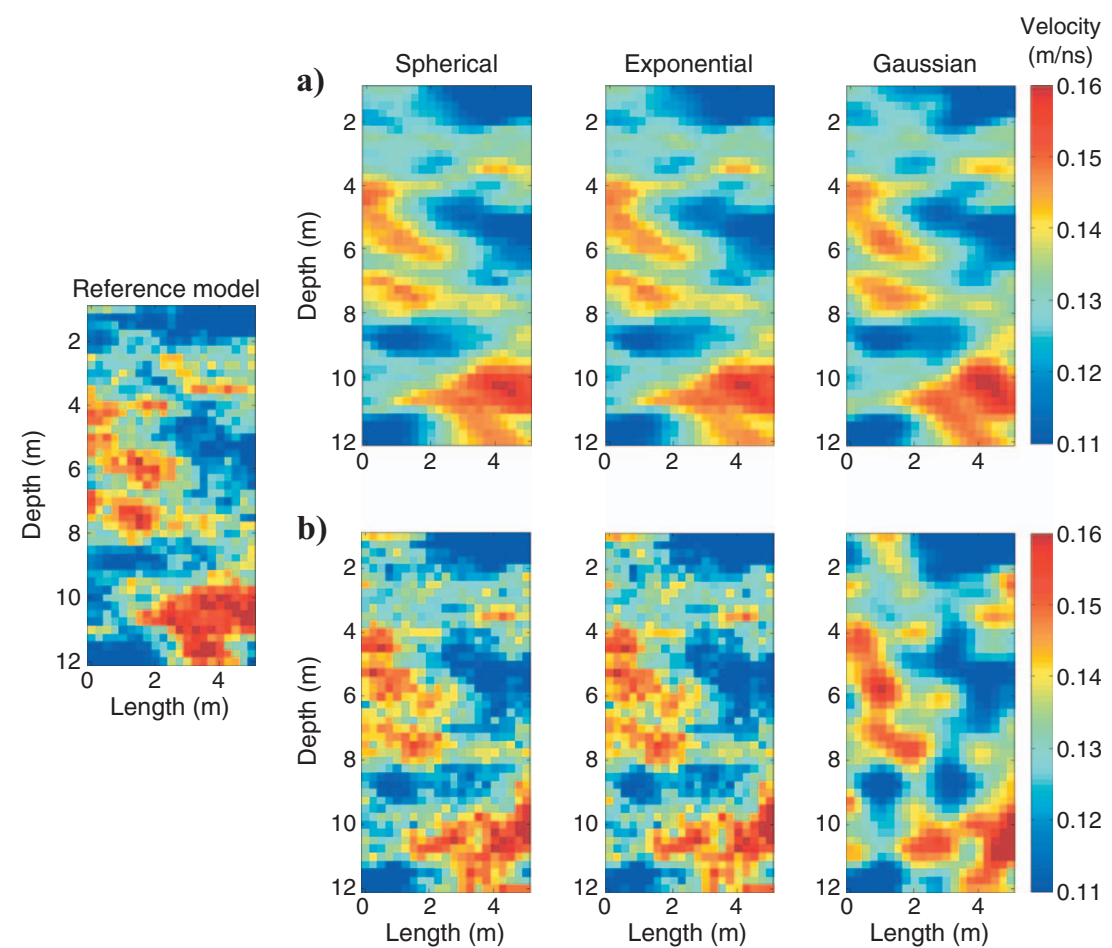

Figure 9. Inversion results assuming different covariance model types - spherical, exponential, and Gaussian - and the corresponding inferred most likely properties obtained in Figure 8. The true subsurface velocity structure is shown to the left. (a) Least-squares inversion estimate. (b) Selected realization drawn from the posterior PDF.
Four multiple-offset gathers (MOGs) were collected during approximately 8 hours, sampling the subsurface below the dotted lines in Figure 10, i.e., borehole pairs GPRA1-3, GPRA2-4, GPRB1-3, and GPRB2-4. The measurements were collected by fixing an antenthe surface and traces having an acquisition angle larger than $45^{\circ}$ were not used in the further analyses. Attenuation of the signal as well as waveguiding along the antennas are known to deteriorate the quality of high-angle traces (Peterson, 2001; Alumbaugh et al., 2002; Irving and Knight, 2005).

Furthermore, the first-arriving EM signal near the surface is refracted waves traveling at the air-soil interface, not the direct wave passing through the subsurface. Because of interference effects between the refracted wave and the direct wave, it is difficult to distinguish between the different waveforms, making estimation of the moisture content at shallow depths problematic. Finally, the relationship between the permittivity and moisture content for the topsoil is likely to differ from that for the sand sequence below, particularly because of the observed clay content. Data from the uppermost $1 \mathrm{~m}$ of the boreholes are therefore omitted from the subsequent tomographic analyses. The resulting data set used for tomographic inversion consisted of 744 traces.

The first-arrival times of the EM waves were picked manually. The velocity distribution was thereafter determined through 2D tomographic inversion to account for all crossing rays passing through each portion of the subsurface. The uncorrelated error level was estimated to be $0.4 \mathrm{~ns}$ from reciprocal measurements (i.e., swapped position of transmitter and receiver antennas), and the correlated error level was set to 2 ns on transmitter and receiver positions according to a study by Cordua et al. (2009). It was necessary to account for correlated data errors to avoid undesired artifacts close to the borehole walls.

\section{Results}

The mean velocity of the subsurface was determined from the traveltime data, and the global variance and the correlation structure were inferred using data-driven ergodic inference. The results are presented in Table 4, and the likelihood distributions are shown in Figure 11.

In Table 4, the estimated mean velocities vary little between the four cross sections and are slightly lower than the mean EM wave velocities estimated from the gravimetric measurements of moisture content. Apart from GPRA2-4 (Table 4), the global variances are also fairly similar and concur well with the gravimetrical estimated value of $0.00025 \mathrm{~m}^{2} / \mathrm{ns}^{2}$ collected in area $\mathrm{B}$. The 
global variance in GPRA2-4 is approximately twice that of the other estimates, a difference also observed in the variance obtained using the collected traveltime data directly.

The inferred correlation lengths range from 1.6 to $6.9 \mathrm{~m}$ in the vertical direction and 0.6 to $6.9 \mathrm{~m}$ in the horizontal direction, being slightly lower in area B. The likelihood plot of GPRB1-3 (Figure $11 \mathrm{~b})$ has many spots with increased likelihood. This could arise if the probed area does not have uniform and stationary geostatistical properties as assumed but instead consists of multiple zones with different spatial characteristics.

A $100-\mathrm{MHz}$ GPR reflection profile conducted during the same week as the crosshole surveys also suggests the presence of structural differences between the two field areas (see Figure 12). The sub-

Table 3. Most likely correlation lengths and corresponding likelihood and data misfit for three assumptions regarding covariance model types.

\begin{tabular}{lcccc} 
Model type & $\begin{array}{c}H_{\max } \\
(\mathrm{m})\end{array}$ & $\begin{array}{c}H_{\min } \\
(\mathrm{m})\end{array}$ & Likelihood & Data misfit \\
\hline Spherical & 3.64 & 1.81 & 0.0104 & $4.71 \times 10^{-4}$ \\
Exponential & 4.33 & 2.87 & 0.0043 & $4.73 \times 10^{-4}$ \\
Gaussian & 1.31 & 1.07 & 0.0032 & $6.54 \times 10^{-4}$ \\
\hline
\end{tabular}
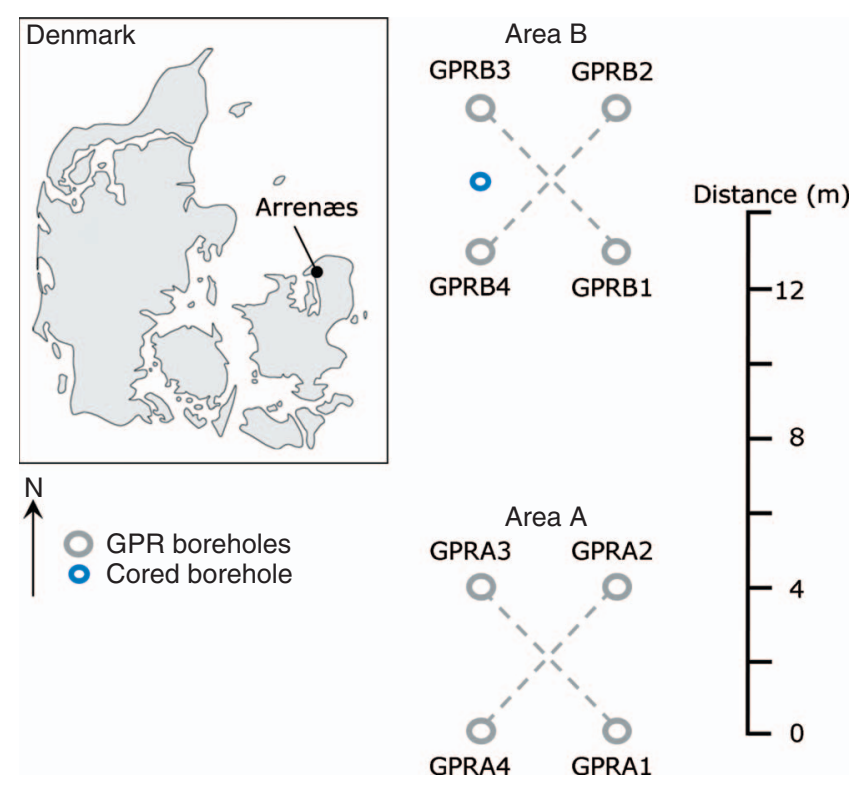

Figure 10. Schematic of the field site setup at Arrenæs, Denmark. surface, sampled in the two field areas, is highlighted with red. The presented GPR reflection profile, running through both field areas, suggests a greater depth penetration $(\sim 15 \mathrm{~m})$ of the EM wave energy toward the south compared with the north $(8-10 \mathrm{~m})$ and a greater density of reflections toward the southern end of the profile. In particular, the reflections in area A appear longer and more continuous, consistent with the longer horizontal correlation lengths at this field area inferred using data-driven ergodic inference. A few reflections appear to be longer than the horizontal correlation length estimated by the inference method. See in particular the reflection around 6 and $8 \mathrm{~m}$ depth in areas A and B, respectively, which is continuous for approximately $40 \mathrm{~m}$. As noted, the applied inference method assumes uniform and stationary geostatistical properties, and the inferred properties are therefore representative of the overall correlation structures of the probed subsurface.

Figure 13 shows the inversion results of the four real data sets using the inferred geostatistical properties. The depth of the reflections in Figure 12 cannot be compared directly to the structures observed in Figure 13 because a constant uniform EM velocity of $0.13 \mathrm{~m} / \mathrm{ns}$ is assumed for the depth conversion in Figure 12. It is apparent from Figure 13 that this assumption is invalid. Nonetheless, at area A, a clear interface in the tomograms is observed at $8 \mathrm{~m}$ depth, and two slightly more indistinct interfaces are found at $4 \mathrm{~m}$ and (albeit weaker) $6 \mathrm{~m}$ depth, matching reflection observations fairly well. In area B (in particular in GPRB2-4), clear interfaces in the tomograms at 8 and $10 \mathrm{~m}$ depth match reflections observed in Figure 12 with a greater precision. In GPRB1-3, these interfaces are not horizontally connected. This results from the inferred horizontal correlation length of just $0.55 \mathrm{~m}$. If a higher value had been used in the inversion, corresponding to another area of high likelihood in Figure 11, the observed features in Figure 13 would be more layered.

In the previous analyses, we assumed a zero dip of the subsurface. It is, however, apparent in Figure 12 that the subsurface structures dip slightly toward the north. To improve the estimates of the geostatistical properties, the dip could be determined within the proposed framework (Hansen et al., 2008) or included as a priori knowledge from, e.g., GPR reflection profiles. The two GPR cross sections collected at each field area could also be combined in a 3D analysis to better constrain the correlation lengths and the dip. However, in a $3 \mathrm{D}$ setup, the number of unknown parameters increases to nine (one extra correlation length and two extra rotational angles), making unique identification of all parameters more challenging.

\section{DISCUSSION}

Our work illustrates the utility of crosshole GPR velocity data to estimate valuable geostatistical information concerning subsurface

Table 4. Data-driven ergodic inference results for four real data sets.

\begin{tabular}{|c|c|c|c|c|c|}
\hline \multirow[b]{2}{*}{ Data set } & \multicolumn{2}{|c|}{ Inferred from traveltime data } & \multicolumn{3}{|c|}{ Data-driven ergodic inference } \\
\hline & $\begin{array}{l}\text { Mean } \\
(\mathrm{m} / \mathrm{ns})\end{array}$ & $\begin{array}{c}\text { Variance } \\
\left(10^{-4} \mathrm{~m}^{2} / \mathrm{ns}^{2}\right)\end{array}$ & $\begin{array}{c}\text { Variance } \\
\left(10^{-4} \mathrm{~m}^{2} / \mathrm{ns}^{2}\right)\end{array}$ & $\begin{array}{l}H_{\min } \\
(\mathrm{m})\end{array}$ & $\begin{array}{c}H_{\max } \\
(\mathrm{m})\end{array}$ \\
\hline GPRA1-3 & 0.1431 & 0.89 & 2.15 & 6.85 & 6.32 \\
\hline GPRA2-4 & 0.1459 & 1.65 & 5.31 & 5.79 & 6.85 \\
\hline GPRB1-3 & 0.1317 & 0.68 & 3.33 & 1.59 & 0.55 \\
\hline GPRB2-4 & 0.1358 & 0.74 & 3.65 & 3.69 & 6.85 \\
\hline
\end{tabular}


structures. Compared with findings of Hansen et al. (2008), our proposed methodology provides a complete geostatistical inference, i.e., an estimation of mean velocity, global variance velocity, and the correlation structures. Because the methodology uses GPR traveltime data indirectly, the geostatistical inference is not affected by smoothing and/or regularization (a limitation stated by Day-Lewis and Lane, 2004).

Synthetic tests show that the mean velocity is determined within a few percent, whereas the global variance velocity can be estimated within a factor of two of the true value. Inference of the studied correlation structures is comparable with results obtained using conventional methods to infer geostatistical properties (e.g., Gstat; Pebesma and Wesseling [1998]). A lower uncertainty of the inferred correlation structures is obtained when subsurface structures are contained within the tomographic image, and the problem of inferring the vertical correlation lengths is better posed because the collected GPR data mainly consist of horizontal traveltime measurements.
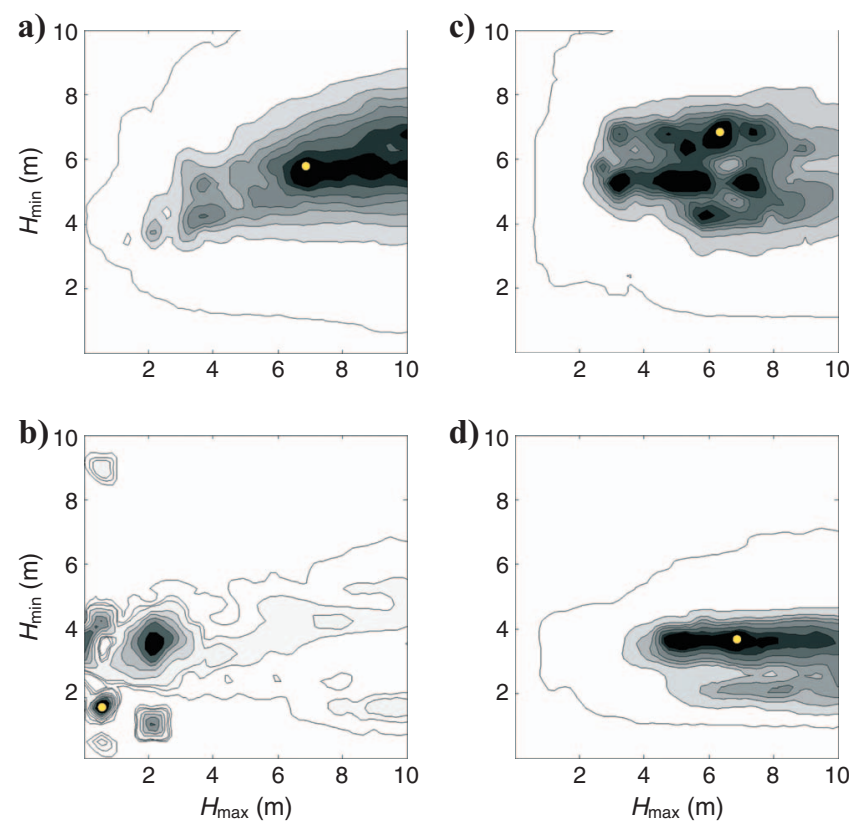

Figure 11. Contour plots showing the likelihood as a function of $H_{\min }$ and $H_{\max }$ obtained using data-driven ergodic inference for four real data sets: (a) GPRA1-3, (b) GPRB1-3, (c) GPRA2-4, and (d) GPRB2-4. The most likely prior covariance models are marked with a yellow dot.

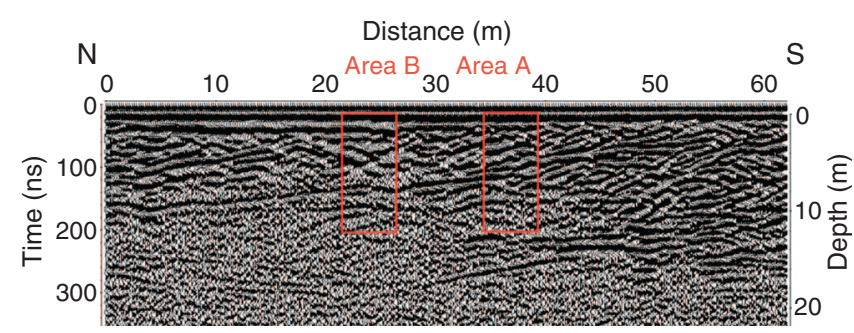

Figure 12. GPR reflection profile collected at Arrenæs intersecting both field areas along a north-south transect. The two red boxes indicate the approximate locations of the two field areas shown in Figure 10 . Vertical depth axis is calculated for a velocity of $0.13 \mathrm{~m} / \mathrm{ns}$. Modified from Hansen et al. (2008).
Irving et al. (2009) have investigated the use of reflection GPR data to infer geostatistical information of the subsurface. The authors expand on findings by Knight et al. (1997) and Rea and Knight (1998) but incorporate the collected GPR data indirectly using an approach somewhat similar to one presented by Hansen et al. (2008). Irving et al. (2009) find that the horizontal correlation lengths are estimated with a high degree of accuracy when the vertical correlation lengths are known. This apparent high sensitivity of reflection GPR data toward the horizontal correlation lengths could, if combined with crosshole GPR data, potentially improve the accuracy of the geostatistical inference method used in our study.

Asli et al. (2000) suggest an alternative method for inferring the covariance model, relying on the comparison of an experimental data covariance model obtained from data observations and a theoretical data covariance calculated from a prior choice of model covariance. This method is used by Gloaguen et al. $(2005,2007)$ and implemented by Giroux et al. (2007). Shamsipour et al. (2010) also apply this method to infer a prior covariance model and describe how applying the method relies on manual intervention such as visual inspection and trial-and-error. Shamsipour et al. (2010) test the methodology on a synthetic test case consisting of data of point support and not volume average data, as considered in our work.

Traveltime data containing a high degree of added noise ( $>0.8 \mathrm{~ns}$ ) adversely affect the geostatistical inference. However, noise levels below $0.8 \mathrm{~ns}$ are certainly feasible, especially if the data set is preprocessed to eliminate errors occurring from incorrect borehole geometry knowledge, positioning errors, and other static errors (as suggested by Squires et al. [1992] and Peterson [2001]). Alternatively, strongly correlated errors could be accounted for during inversion using an approach similar to the one used by Cordua et al. (2009).

In our work investigating whether our proposed framework could be used to identify which covariance function provides the best description of the subsurface variability, the correct covariance function (in the investigated case, a spherical model) had a slightly higher
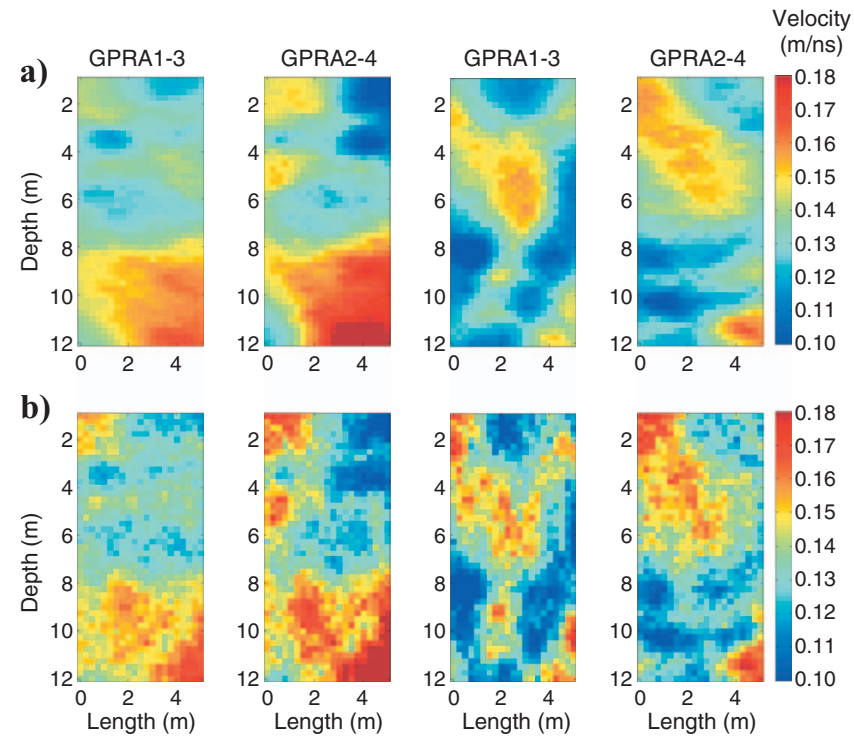

Figure 13. Inversion results using data-driven ergodic inference for four real data sets: GPRA1-3, GPRA2-4, GPRB1-3, and GPRB2-4. (a) The least-squares inversion estimate. (b) A selected realization drawn from the posterior PDF. 
likelihood than the other two investigated covariance functions (Gaussian and exponential). But the question as to whether this difference is significant enough to provide unique estimations has not been addressed. Instead, we have shown that assuming an erroneous covariance model type does not necessarily affect the obtained inversion results. In such a case, representative correlation lengths were estimated and the least-squares estimate and realizations of the posterior PDF therefore were not affected adversely.

Although our emphasis has been the application of ergodic inference to crosshole GPR tomography, we stress that ergodic inference, as presented here, is applicable to any least-squares-based inversion problem relying on a Gaussian a priori model, such as described by Tarantola and Valette (1982).

\section{CONCLUSION}

We have evaluated and expanded on a methodology (i.e., datadriven ergodic inference) to infer geostatistical properties of the unsaturated zone using crosshole GPR data alone. Based on these new results, we conclude that the properties are inferred without using the inversion images directly. Excessive smoothing/damping often observed in previous literature therefore do not affect the result.

The inferred geostatistical properties have two main applications:

1) An accurate $\mathbf{C}_{m}$ improves the reliability of the inversion results. We illustrated the large effect that the choice of prior covariance model has on the results of stochastic inversion. A proper selection of the prior covariance model is therefore paramount to ensure the validity of the realizations drawn from the posterior PDF.

2) Geostatistical properties describe the entire subsurface also the unprobed area and can therefore be used as input in stochastic hydrologic models.

The geostatistical properties inferred using data-driven ergodic inference will in every case improve the inversion results (application 1) because the inferred properties honor the collected traveltime data by having the highest likelihood. Caution should, however, be taken for application 2. Our proposed method worked satisfactorily for correlation lengths contained within or slightly exceeding the model domain, but the inferred geostatistical properties should not be expected to represent the true subsurface structures when the correlation structures are predominantly larger than the area of measurement (i.e., interborehole area).

Finally, we must stress that our methodology is not restricted to crosshole GPR data collected in the unsaturated zone but could be used for a wide range of data types and other purposes. Crosshole GPR data collected in the saturated zone could be used to infer the geostatistical properties of the porosity distribution of a given porous media, a physical property that, in some cases, may be correlated to the hydraulic conductivity and storage capacities of the media.

\section{ACKNOWLEDGMENTS}

We wish to thank the Water Department, Section for Water Quality, at Copenhagen Energy for all their help and for use of their field location at the Arrenæs infiltration plant. Furthermore, M. Looms thanks the many helpers who assisted her in the field data collection. This work was completed while M. Looms was employed at HOBE, Center of Hydrology — Hydrological Observatory. During much of this study, T. M. Hansen was financed by a Faculty Research Grant from the Faculty of Science, University of Copenhagen.

\section{REFERENCES}

Alumbaugh, D., P. Y. Chang, L. Paprocki, J. R. Brainard, R. J. Glass, and C. A. Rautman, 2002, Estimating moisture contents in the vadose zone using cross-borehole ground penetrating radar: A study of accuracy and repeatability: Water Resources Research, 38, 1309-1321, doi: 10.1029/ 2001WR000754.

Annan, A. P., 2005, GPR methods for hydrogeological studies, in Y. Rubin and S. S. Hubbard, eds., Hydrogeophysics: Springer, 129-156.

Asli, M., D. Marcotte, and M. Chouteau, 2000, Direct inversion of gravity data by cokriging: 6 th International Geostatistics Congress, Proceedings. 64-73.

Binley, A., G. Cassiani, and P. Winship, 2004, Characterization of heterogeneity in unsaturated sandstone using borehole logs and cross-borehole tomography, in J. S. Bridge and D. W. Hyndman, eds., Aquifer characterization: Society for Sedimentary Geology Special Publication, 80, 129-138.

Binley, A., P. Winship, R. Middleton, M. Pokar, and J. West, 2001, High-resolution characterization of vadose zone dynamics using cross-borehole radar: Water Resources Research, 37, 2639-2652, doi: 10.1029/ 2000WR000089.

Constable, S. C., R. L. Parker, and C. G. Constable, 1987, Occam's inversion: A practical algorithm for generating smooth models from electromagnetic sounding data: Geophysics, 52, 289-300, doi: 10.1190/1.1442303.

Cordua, K. S., M. C. Looms, and L. Nielsen, 2008, Accounting for correlated data errors during inversion of cross-borehole ground penetrating radar data: Vadose Zone Journal, 7, no. 1, 263-271, doi: 10.2136/vzj2007.0008.

Cordua, K. S., L. Nielsen, M. C. Looms, T. M. Hansen, and A. Binley, 2009, Quantifying the influence of static-like errors in least-squares-based inversion and sequential simulation of cross-borehole ground penetrating radar data: Journal of Applied Geophysics, 68, no. 1, 71-84, doi: 10.1016/j.jappgeo.2008.12.002.

Dafflon, B., J. Irving, and K. Holliger, 2009, Use of high-resolution geophysical data to characterize heterogeneous aquifers: Influence of data integration method on hydrological predictions: Water Resources Research, $\mathbf{4 5}$, no. 9, W09407, doi: 10.1029/2008WR007646.

Davis, J. L., and A. P. Annan, 1989, Ground-penetrating radar for high-resolution mapping of soil and rock stratigraphy: Geophysical Prospecting, 37, no. 5, 531-551, doi: 10.1111/j.1365-2478.1989.tb02221.x.

Day-Lewis, F. D., and J. W. Lane, Jr., 2004, Assessing the resolution-dependent utility of tomograms for geostatistics: Geophysical Research Letters, 31, no. 7, L07503, doi: 10.1029/2004GL019617.

Day-Lewis, F. D., K. Singha, and A. M. Binley, 2005, Applying petrophysical models to radar travel time and electrical resistivity tomograms: Resolution-dependent limitations: Journal of Geophysical Research, 110, B8, B08206, doi: 10.1029/2004JB003569.

Eppstein, M. J., and D. E. Dougherty, 1998, Efficient three-dimensional data inversion: Soil characterization and moisture monitoring from cross-well ground penetrating radar at a Vermont test site: Water Resources Research, 34, 1889-1900, doi: 10.1029/98WR00776.

Ernst, J. R., A. G. Green, H. Maurer, and K. Holliger, 2007, Application of a new 2D time-domain full-waveform inversion scheme to crosshole radar data: Geophysics, 72, no. 5, J53-J64, doi: 10.1190/1.2761848.

Ernst, J. R., K. Holliger, H. Maurer, and A. G. Green, 2006, Realistic FDTD modelling of borehole georadar antenna radiation: Methodology and application: Near Surface Geophysics, 4, no. 1, 19-30.

Ferré, P. A., D. L. Rudolph, and R. G. Kachanoski, 1996, Spatial averaging of water content by time domain reflectometry: Implications for twin rod probes with and without dielectric coatings: Water Resources Research, 32, no. 2, 271-279, doi: 10.1029/95WR02576.

Giroux, B., E. Gloaguen, and M. Chouteau, 2007, bh_tomo - A Matlab borehole georadar 2D tomography package: Computers \& Geosciences, 33, no. 1, 126-137, doi: 10.1016/j.cageo.2006.05.014.

Gloaguen, E., B. Giroux, D. Marcotte, and R. Dimitrakopoulos, 2007, Pseudo-full-waveform inversion of borehole GPR data using stochastic tomography: Geophysics, 72, no. 5, J43-J51, doi: 10.1190/1.2755929.

Gloaguen, E., D. Marcotte, M. Chouteau, and H. Perroud, 2005, Borehole radar velocity inversion using cokriging and cosimulation: Journal of Applied Geophysics, 57, no. 4, 242-259, doi: 10.1016/j.jappgeo.2005.01.001.

Goovaerts, P., 1997, Geostatistics for natural resources evaluation: Oxford University Press.

Hansen, T. M., and K. Mosegaard, 2008, VISIM: Sequential simulation for linear inverse problems: Computers \& Geosciences, 34, no. 1, 53-76, doi: 10.1016/j.cageo.2007.02.003

Hansen, T. M., A. G. Journel, A. Tarantola, and K. Mosegaard, 2006, Linear inverse Gaussian theory and geostatistics: Geophysics, 71, no. 6, R101-R111, doi: 10.1190/1.2345195. 
Hansen, T. M., M. C. Looms, and L. Nielsen, 2008, Inferring a sub-surface structural covariance model using cross-borehole ground penetrating radar tomography: Vadose Zone Journal, 7, no. 1, 249-262, doi: 10.2136/ vzj2006.0144.

Hubbard, S. S., J. E. Peterson, J. Roberts, and F. Wobber, 1997, Estimation of permeable pathways and water content using tomographic radar data: The Leading Edge, 16, 1623-1628, doi: 10.1190/1.1437539.

Hubbard, S. S., Y. Rubin, and E. Majer, 1999, Spatial correlation structure estimation using geophysical and hydrogeological data: Water Resources Research, 35, 1809-1825, doi: 10.1029/1999WR900040.

Irving, J. D., and R. J. Knight, 2005, Effect of antennas on velocity estimates obtained from cross-borehole GPR data: Geophysics, 70, no. 5, K39-K42, doi: 10.1190/1.2049349.

, 2006, Numerical modeling of ground-penetrating radar in 2-D using MATLAB: Computers \& Geosciences, 32, no. 9, 1247-1258, doi: 10.1016/j.cageo.2005.11.006.

Irving, J., R. Knight, and K. Holliger, 2009, Estimation of the lateral correlation structure of subsurface water content from surface-based ground-penetrating radar reflection images: Water Resources Research, 45, no. 12 , W12404, doi: 10.1029/2008WR007471

Journel, A. G., and C. J. Huijbregts, 1978, Mining geostatistics: Academic Press.

Knight, R., P. Tercier, and H. Jol, 1997, The role of ground penetrating radar and geostatistics in reservoir description: The Leading Edge, 16, 1576-1582, doi: 10.1190/1.1437526.

Kowalsky, M. B., S. Finsterle, J. Peterson, S. Hubbard, Y. Rubin, E. Majer, A. Ward, and G. Gee, 2005, Estimation of field-scale soil hydraulic and dielectric parameters through joint inversion of GPR and hydrological data: Water Resources Research, 41, no. 11, W11425, doi: 10.1029/ 2005WR004237.

Linde, N., A. Binley, A. Tryggvason, L. B. Pedersen, and A. Revil, 2006, Improved hydrogeophysical characterization using joint inversion of crosshole electrical resistance and ground penetrating radar traveltime data: Water Resources Research, 42, no. 12, W12404, doi: 10.1029/ 2006WR005131.

Looms, M. C., K. H. Jensen, A. Binley, and L. Nielsen, 2008, Monitoring unsaturated flow and transport using cross-borehole geophysical methods:
Vadose Zone Journal, 7, no. 1, 227-237, , doi: 10.2136/vzj2006.0129.

Marquardt, D. W., 1970, Generalized inverses, ridge regression, biased linear estimation and non-linear estimation: Technometrics, 12, 591-612, doi: $10.2307 / 1267205$.

Menke, W., 1989, Geophysical data analysis: Discrete inverse theory: Academic Press Inc.

Pardo-Igúzquiza, E., 1998, Maximum likelihood estimation of spatial covariance parameters: Mathematical Geology, 30, no. 1, 95-108, doi: 10.1023/A:1021765405952.

Pebesma, E. J., and C. G. Wesseling, 1998, Gstat: A program for geostatistical modelling, prediction and simulation: Computers \& Geosciences, 24, no. 1, 17-31, doi: 10.1016/S0098-3004(97)00082-4.

Peterson, J. E., Jr., 2001, Pre-inversion corrections and analysis of radar tomographic data: Journal of Environmental \& Engineering Geophysics, 6, no. 1, 1-18, doi: 10.4133/JEEG6.1.1.

Rea, J., and R. Knight, 1998, Geostatistical analysis of ground-penetrating radar data: A means of describing spatial variation in the subsurface: Water Resources Research, 34, no. 3, 329-339, doi: 10.1029/97WR03070.

Shamsipour, P., D. Marcotte, M. Chouteau, and P. Keating, 2010, 3D stochastic inversion of gravity data using cokriging and cosimulation: Geophysics, 75, no. 1, I1-I10, doi: 10.1190/1.3295745.

Squires, L. J., S. N. Blakeslee, and P. L. Stoffa, 1992, The effects of statics on tomographic velocity reconstructions: Geophysics, 57, 353-362, doi: 10.1190/1.1443249.

Tarantola, A., 1987, Inverse problem theory - Methods for data fitting and model parameter estimation: Elsevier Science Publ. Co., Inc.

Tarantola, A., and B. Valette, 1982, Generalized nonlinear inverse problems solved using the least squares criterion: Reviews of Geophysics and Space Physics, 20, no. 2, 219-232, doi: 10.1029/RG020i002p00219.

Topp, G. C., J. L. Davis, and A. P. Annan, 1980, Electromagnetic determination of soil water content: Measurements in coaxial transmission lines: Water Resources Research, 16, 574-582, doi: 10.1029/ WR016i003p00574.

Zelt, C. A., and P. J. Barton, 1998, Three-dimensional seismic refraction tomography: A comparison of two methods applied to data from the Faeroe Basin: Journal of Geophysical Research, 103, 7187-7210, doi: 10.1029/ 97JB03536. 